\title{
ANALYSIS OF EVENT RELATED POTENTIALS USING PCA AND MATCHING PURSUIT ON THE TIME-FREQUENCY PLANE
}

Selin Aviyente

\author{
Edward M. Bernat, Stephen M. Malone, William G. Iacono
}

\begin{abstract}
Joint time-frequency representations offer a rich representation of event related potentials (ERPs) that cannot be obtained through individual time or frequency domain analysis. This rich representation, however, comes at the expense of increased data volume and the difficulty of interpreting the resulting representations. Therefore, methods that can reduce the large amount of time-frequency data to physiologically meaningful components are essential. The method presented in this paper extends the well-known principal component analysis to the time-frequency plane to reduce a large set of ERPs to a small number of significant components. These components are then characterized using a matching pursuit algorithm with a gabor dictionary to offer a succinct parametrization of the ERP data. The results show that the principal component analysis is successful at extracting components that can be described as the superposition of a small number of gabor logons, and that the resulting set of parameters succinctly represent physiologically meaningful ERP events.
\end{abstract}

\section{INTRODUCTION}

Event related potentials are transient signals which appear as voltage deviations in the electroencephalogram (EEG) and are caused by external stimuli or cognitive processes triggered by external events. ERPs like most other real life signals are nonstationary and thus can be best tackled by using nonstationary signal analysis techniques. Some common analysis tools include wavelet transform, matching pursuit analysis and Cohen's class of time-frequency distributions (TFDs) [1, 2, 3]. Cohen's class of distributions provides advantages over the other time-frequency representations such as accurate characterization of the physical timefrequency properties of a signal, e.g., energy and marginals, and yields uniform high resolution over the time-frequency plane. This high resolution representation comes at the expense of increased data and the difficulty of interpreting the resulting three-dimensional surfaces. For this reason, data reduction techniques are needed to extract physiologically meaningful information from TFD surfaces.

Current time-frequency data reduction methods involve computing energy over frequency subbands, choosing the best subbands in wavelet packet decomposition or using sparse representations such as matching pursuit (e.g., $[4,5])$. The major shortcoming of these methods is that they assume $a$ priori signal model and reduce the time-frequency information based on this model. Recently, a data-driven method based on principal component analysis (PCA) combined with varimax rotation has been proposed to decompose the ERP time-frequency surfaces and reduce the large amount of data into a few significant principal components [6]. This method was shown to extract ERP components that are localized on the time-frequency plane and that correspond to well-known physiological phenomena.

In this paper, we propose an extension of this PCAbased method to further reduce the information from the principal components into fewer parameters. Since the principal components extracted from ERP time-frequency surfaces are well-localized in time and frequency, we propose quantifying them in terms of well-known compact signals on the time-frequency plane. Gabor logons are known to achieve the lower bound of the uncertainty principle on the time-frequency plane and are well-equipped to explain the time and frequency modulations in a signal [7]. For this reason, we propose to decompose the principal components in terms of time-frequency shifted and scaled gabor logons. An overcomplete dictionary of gabor logons is formed and an algorithm similar to matching pursuit is developed on the time-frequency plane to determine the number of logons necessary to describe an ERP component [5]. This combined approach provides a way of characterizing ERPs in terms of a few time-frequency parameters and offers a powerful tool for extracting a succinct representation of information from large data sets.

The rest of this paper is organized as follows. Section 2 outlines the principal component analysis as applied to time-frequency distributions. Section 3 introduces a match- 
ing pursuit type algorithm as adapted to time-frequency distributions. This analysis enables us to parametrize the principal components in terms of gabor logons. Section 4 discusses the results for both a set of ERP signals and synthesized signals. Finally, Section 5 concludes the paper and discusses the major contributions.

\section{PCA ON THE TIME-FREQUENCY PLANE}

Ideally, an ERP TFD data reduction method will faithfully reproduce established time-based findings (i.e. peaks in the time domain such as P300 or summaries of frequency activity such as alpha), but also allow a more complex view of these phenomena using the rich information available in the TFDs. PCA is widely used for ERP data reduction, and few alternatives offer its power and flexibility as a data driven method for characterizing activity. The decomposition method used in this paper is based on a direct extension of PCA as employed in the time or frequency domains separately, into the time-frequency domain and can be summarized as follows:

1. Compute the time-frequency distribution of each ERP waveform.

$$
\begin{aligned}
T F D(n, \omega ; \psi) & =\sum_{n_{1}=-N}^{N} \sum_{n_{2}=-N}^{N} x\left(n+n_{1}\right) x^{*}\left(n+n_{2}\right) \\
& \times \psi\left(-\frac{n_{1}+n_{2}}{2}, n_{1}-n_{2}\right) e^{-j \omega\left(n_{1}-n_{2}\right)},
\end{aligned}
$$

where $\psi$ is the time-frequency kernel and $x(n)$ is the ERP waveform.

2. Given $N$ ERP waveforms, rearrange the time-frequency surfaces into vectors and form the matrix

$$
\mathbf{X}=\left[\begin{array}{c}
\mathbf{T F D}_{1}^{T} \\
\mathbf{T F D}_{2}^{T} \\
\vdots \\
\mathbf{T F D}_{N}^{T}
\end{array}\right]
$$

3. Compute the covariance matrix $\boldsymbol{\Sigma}=\mathbf{X X}^{\mathbf{T}}$.

4. Decompose the covariance matrix using principal component analysis.

$$
\boldsymbol{\Sigma}=\sum_{i=1}^{N} \lambda_{i} \mathbf{P C}_{i} \mathbf{P} \mathbf{C}_{i}^{T}
$$

where $\lambda_{i}$ is the eigenvalue of each principal component $\mathbf{P C}_{i}$. The principal components determine the span of the time-frequency space.
5. Rotate the principal components using varimax rotation [8]. Varimax rotation is an orthogonal transform that rotates the principal components such that the variance of the factors is maximized. This rotation improves the interpretability of the principal components.

6. Rearrange each principal component into a time-frequency surface to obtain the ERP components in the timefrequency domain.

\section{MATCHING PURSUIT ON THE TIME-FREQUENCY PLANE}

In this section, we introduce a matching pursuit type algorithm on the time-frequency plane to further reduce the time-frequency data into a few significant parameters. The goal is to be able to determine the complexity of the principal component surfaces and to be able to describe them using a compact set of time-frequency features. It is known that the gabor logon, a gaussian window modulated in frequency, is the most compact signal on the time-frequency plane as shown by the uncertainty principle. Therefore, when quantifying the compactness of a given signal on the time-frequency plane, it is natural to take the gabor logons as the building blocks. We propose a time-frequency decomposition method in terms of gabor logons to determine the complexity of principal component surfaces and to further parameterize them. The proposed algorithm is similar to the original matching pursuit [5] and the discrete Gabor decomposition [9], except for the fact that it is developed in the time-frequency domain rather than in the time domain.

A dictionary of gabor logons on the time-frequency plane is constructed and the decomposition is performed using the elements of this dictionary, $G\left(n, \omega ; n_{0}, \omega_{0}, \alpha\right)$ :

$$
G(n, \omega)=\exp \left(-\alpha\left(n-n_{0}\right)^{2}\right) \exp \left(-\frac{\left(\omega-\omega_{0}\right)^{2}}{\alpha}\right),
$$

where $\alpha$ is the scale parameter, $n_{0}$ and $\omega_{0}$ are the time and frequency shift parameters, respectively. The decomposition algorithm for a given TFD can be summarized as follows:

1. Initialize the residue as $T F D_{0}=T F D$, and the iteration index $k=1$.

2. Find the gabor logon over the whole dictionary, i.e. over all $\left(n_{0}, \omega_{0}, \alpha\right)$, that has the largest inner product with the given time-frequency surface.

$$
G_{k}(n, \omega)=\operatorname{argmax}_{i} \sum_{n} \sum_{\omega} T F D_{k-1}(n, \omega) G_{i}(n, \omega)
$$


3. Subtract the selected gabor logon from the residue to compute the new residue time-frequency distribution.

$$
\begin{array}{r}
T F D_{k}(n, \omega)=T F D_{k-1}(n, \omega) \\
-\left(\sum_{n} \sum_{\omega} T F D_{k-1}(n, \omega) G_{k}(n, \omega)\right) G_{k}(n, \omega)
\end{array}
$$

4. Increment $k$ by 1 .

5. Go back to step 2 until the normalized energy in the residue is below a pre-determined threshold, i.e.,

$$
\frac{\left\|T F D_{k}\right\|_{2}}{\|T F D\|_{2}}<\gamma
$$

\section{RESULTS}

The proposed method of combined principal component analysis and matching pursuit on the time-frequency plane is applied to a set of synthesized signals as well as ERP signals.

\subsection{Data Set}

Three different types of signals are synthesized to illustrate the effect of the algorithm: a mixture of three gabor logons, a mixture of three gabor logons in white noise $(\mathrm{SNR}=4 \mathrm{~dB})$ and a mixture of linear and quadratic chirp signals. First, the principal components with the highest eigenvalues are extracted on the time-frequency plane. Then, the matching pursuit algorithm using a dictionary of gabor logons is applied. The number of gabor logons required to express each principal component at a given energy level is found through the matching pursuit algorithm described in Section 3. The energy level determines how many logons are required to represent a certain percentage of the original time-frequency distribution and is defined in Step 5 of the algorithm.

The ERP data used for analysis consists of participants in the Minnesota Twin Family Study (MTFS), a longitudinal and epidemiological investigation of the origins and development of substance use disorders and related psychopathology. This sample combines subjects from two age cohorts of the MTFS: subjects in one cohort were 17 years old at intake whereas subjects in the other were approximately 11 years old at intake, with data for the latter coming from the second three-year follow-up assessment. Data from 2069 participants (979 male) are assessed. ERP data was recorded during an oddball task in which simple ovals served as frequent stimuli (two-thirds of the trials) and stylized heads served as target stimuli (one-third of the trials). Presented data contain principal components characterizing activity for target stimuli.

\begin{tabular}{|c|c|c|c|}
\hline Signal Type & $95 \%$ Energy & $97 \%$ Energy & $98 \%$ Energy \\
\hline ERP $(0-5.75 \mathrm{~Hz})$ & 1 & 2.19 & 6 \\
\hline ERP $(0-14 \mathrm{~Hz})$ & 1 & 1.14 & 2.64 \\
\hline 3 Logons & 1 & 1.33 & 3.33 \\
\hline 3 Logons in Noise & 1 & 1.33 & 9 \\
\hline Mixture of Chirps & 1 & 1.5 & 34.5 \\
\hline
\end{tabular}

Table 1. Comparison of different signals in terms of the number of logons required to represent them at different energy levels

\subsection{Analysis}

Table 1 summarizes the average number of logons required to represent the principal components for three different energy levels. It can be seen that for all signal classes one gabor logon is enough to express the $95 \%$ of the energy of the extracted principal components. Therefore, the $95 \%$ energy criterion is not significant enough to determine how close the underlying component is to a gabor logon. When the energy level is increased to $98 \%$, it can be seen that the principal components extracted from the synthesized gabor logons are still accurately modeled by a small number of logons whereas the principal components for the other synthesized signals require a larger number of logons. This is mainly due to the fact that once the first logon is subtracted from the distribution, the residue is no longer close to a logon and it requires a combination of logons to represent the remaining energy.

For the ERP signals, two frequency bands are analyzed $(0-5.75 \mathrm{~Hz})$ and $(0-14 \mathrm{~Hz}) .16$ and 14 principal components are analyzed respectively for the $0-5.75 \mathrm{~Hz}$ and the $0-14 \mathrm{~Hz}$ bands. The number of logons required to model the principal component surfaces average to 6 for $0-5.75 \mathrm{~Hz}$ band due to reduced resolution, whereas the same average is 2.64 for $0-14 \mathrm{~Hz}$ band at $98 \%$ energy level. This is still a very small number of logons given the number of elements in the dictionary, 6060. This analysis shows that the ERP TFD surfaces can be well-described by a small set of time-frequency parameters and that the principal component analysis combined with varimax rotation extracts components that are well-localized on the time-frequency plane.

Fig. 1 illustrates the single gabor logons extracted for the 14 principal components for the $0-14 \mathrm{~Hz}$ frequency band. It is computed that the extracted single logons have an average correlation of 0.6394 with the principal components. It is seen that the extracted logons can separate between the early and the late activities, as well as the low frequency activity ( $4 \mathrm{~Hz}$ ) and alpha activity ( $10 \mathrm{~Hz}$ ). Similarly, Fig. 2 illustrates the single gabor logons extracted for the $0-5.75 \mathrm{~Hz}$ frequency band. As before, the extracted gabor logons separate the activity at the low frequency bands $(0-2 \mathrm{~Hz})$ from the higher frequency band $(4-5.75 \mathrm{~Hz})$. 

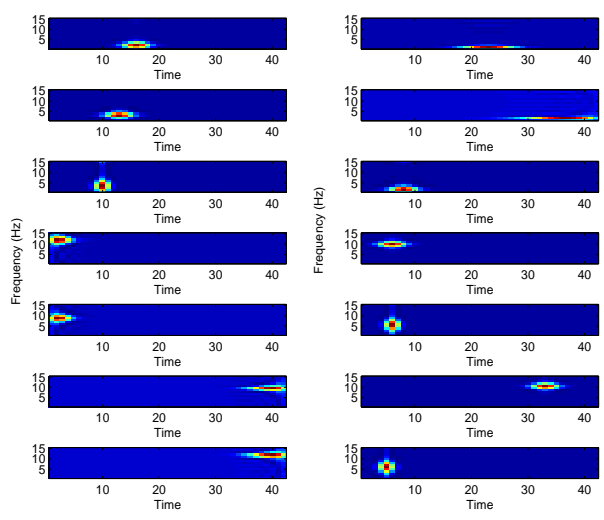

Fig. 1. The single gabor logons that are extracted from the first 14 principal components of the ERP dataset $(0-14 \mathrm{~Hz})$
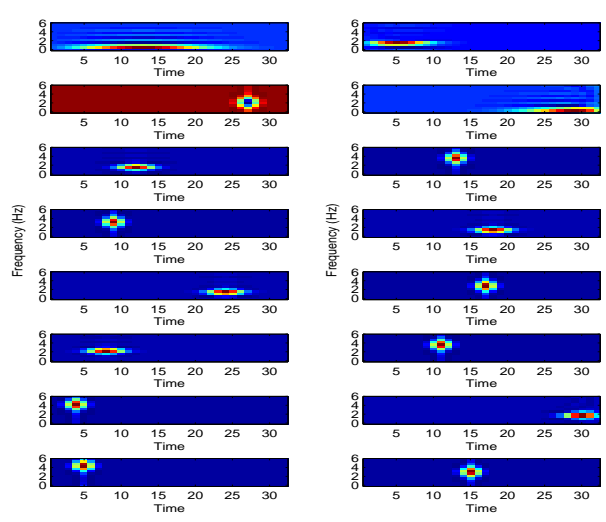

Fig. 2. The single gabor logons that are extracted from the first 16 principal components of the ERP dataset $(0-5.75 \mathrm{~Hz})$

\section{CONCLUSIONS}

In this paper, a data reduction method based on principal component analysis combined with matching pursuit was presented. Using the proposed method, it was possible to extract components from simulated and ERP data that are well-characterized by a small number of gabor logons. This characterization offers several contributions. First, it suggests that the principal components approach employed in the first stage of the current method tends to extract gabor logons. Because gabor logons are the most compact timefrequency signal representations, it can be inferred that under the tested circumstances the principal components were an optimal signal representation. Second, the gabor parametrization is a more succinct representation of the energy than the complete time-frequency principal component surfaces, and thus offers greater power for analysis. Another significance of the proposed method is that unlike other data reduction methods on the time-frequency plane such as matching pursuit or chirp transform, the proposed method does not make any assumptions about the underlying signals, i.e. it does not try to fit a pre-determined signal model to the given data. Finally, current findings suggest that the alpha activity characterized in the ERP signals operates in a manner consistent with gabor logons. This extends previous work by detailing the nature of the event-related desynchronization (ERD) of the alpha during cognitive processing, and the event-related synchronization (ERS) as alpha power returns post-processing.

Future work can evaluate these gabor parameters in relation to well-known cognitive ERP events such as P300, as well as ERP events with known specific physiological origins, such as anterior cingulate cortex activation as measured in the error-related negativity (ERN) paradigm.

\section{REFERENCES}

[1] T. Demiralp, J. Yordanova, V. Kolev, A. Ademoglu, M. Devrim, and V. J. Savar, "Time-frequency analysis of single sweep event-related potentials by means of fast wavelet transform," Brain and Language, vol. 66, pp. 129-145, 1999.

[2] P. J. Durka and K. J. Blinowska, "A unified timefrequency parametrization of EEGs," IEEE Engineering in Medicine and Biology, vol. 20, no. 5, pp. 47-53, September/October 2001.

[3] W. J. Williams, "Reduced interference distributions: Biological applications and interpretations," Proceedings of the IEEE, vol. 84, no. 9, pp. 1264-1280, Sept. 1996.

[4] R. R. Coifman and M. V. Wickerhauser, "Entropybased algorithms for best basis selection," IEEE Trans. on Info. Theory, vol. 38, no. 2, pp. 713-718, 1992.

[5] S. Mallat and Z. Zhang, "Matching pursuits with timefrequency dictionaries," IEEE Trans. on Signal Processing, vol. 41, pp. 3397-3415, 1993.

[6] E. M. Bernat, W. J. Williams, and W. J. Gehring, "Decomposing ERP time-frequency energy using PCA," Clinical Neurophysiology, vol. 116, pp. 1314-1334, 2005.

[7] L. Cohen, Time-Frequency Analysis, Prentice Hall, New Jersey, 1995.

[8] H. F. Kaiser, "The varimax criterion for analytic rotation in factor analysis," Psychometrika, vol. 23, pp. 187-200, 1958. 
[9] S. Qian and D. Chen, "Discrete gabor transform," IEEE

Trans. on Signal Processing, vol. 41, no. 7, 1993. 